\title{
Neuroendocrine liver metastases treatment strategies: a viewpoint
}

\author{
Roberta Elisa Rossi ${ }^{1,2}$ \\ ${ }^{1}$ Department of Hepatology, Hepato Pancreatic Biliary, Surgery and Liver Transplantation, ENETS Center of Excellence, Fondazione IRCCS \\ Istituto Nazionale Tumori (INT, National Cancer Institute), Milan, Italy; ${ }^{2}$ Department of Pathophysiology and Transplantation, University of Milan, \\ Milan, Italy \\ Correspondence to: Roberta Elisa Rossi. Department of Hepatology, Hepato Pancreatic Biliary, Surgery and Liver Transplantation, ENETS Center of \\ Excellence, Fondazione IRCCS Istituto Nazionale Tumori (INT, National Cancer Institute), Milan, Italy. Email: robertaelisa.rossi@gmail.com. \\ Comment on: Cloyd JM, Ejaz A, Konda B, et al. Neuroendocrine liver metastases: a contemporary review of treatment strategies. Hepatobiliary Surg \\ Nutr 2020;9:440-51.
}

Submitted May 20, 2021. Accepted for publication Jun 03, 2021.

doi: $10.21037 / \mathrm{hbsn}-21-178$

View this article at: https://dx.doi.org/10.21037/hbsn-21-178

I read with great interest the article "Neuroendocrine liver metastases: a contemporary review of treatment strategies" (1) published in Hepatobiliary Surgery and Nutrition as it is focused on a debate topic.

The majority of gastro-entero-pancreatic neuroendocrine neoplasms (GEP-NENs) metastasize to the liver, being the presence of liver metastases (NELM) a negative prognostic factor. There is still controversy regarding the optimal management of patients with NELM.

Surgery plays a key-role role as, indeed, the resection of the primary tumor and its metastases remains, when possible, the only curative treatment in patients with GEPNENs. Surgical resection with curative intent is reserved to patients with no or minimum extra-hepatic disease and with limited tumor burden and, even if no randomized controlled trials are available comparing surgery versus non-surgical therapies, resection of NELM is reported to be associated with excellent long-term outcomes and should be the preferred treatment option for patients with NELM (1).

Although still controversial, debulking surgery is suggested if at least $80-90 \%$ of tumor load can be removed with promising results in terms of both overall survival (OS), with a reported 5 -year OS of $60 \%$, and symptom control; some authors have also proposed to modify the threshold for considering cytoreductive surgery to a $70 \%$ threshold (1).

In metastatic cases not amenable to surgery because of disease's extent or patient's co-morbidities, liver-directed therapies represent a viable option to improve progressionfree survival (PFS) and to control hormonal symptoms. However, prospective studies comparing the different trans- arterial treatment strategies are scanty and decisions regarding liver-directed therapies have traditionally been related to institutional preferences and local expertise. Several medical options are also available for advanced metastatic NENs and these include somatostatin analogs (SSAs) as first-line treatment for tumor growth and symptom's control, targeted therapies (i.e., everolimus and sunitinib), chemotherapy mainly for G3 neoplasms and pancreatic forms and peptide receptor radionuclide therapy (PRRT), which demonstrated improved PFS compared with high-dose SSA among patients with midgut NENs and has been repeatedly reported as particularly useful for symptom relief in functioning forms.

In highly selected cases ( $<1 \%)$, liver transplant (LT) might be considered in patients with NELM representing a potential chance of radical cure if proper patient selection is provided. Satisfactory long-term outcomes have been reported and, in the only available comparative study, a survival benefit of nearly 3.5 years at 10 years in favor of LT versus non-transplant strategies was observed (2). However, results are still heterogeneous with some studies suggesting similar survival outcomes among patients within Milan criteria who undergo surgical resection compared to LT (3). Although post-LT recurrence is observed in $30-50 \%$ of the cases, excellent long-term survival is observed after postLT NEN recurrence, especially when recurrence appears late after transplant, and an aggressive surgical treatment might result in a new chance of cure for selected patients (4). Considered the favourable long-term outcomes after LT, some authors suggested to adopt criteria that are less restrictive to not deny the patient's access to a definitive 
treatment. With this regard, the identification of multianalyte markers, including, microRNA, Next Generation Sequencing (NGS) analysis and test, detecting minimal extrahepatic neuroendocrine disease, which is responsible for late recurrences, should be encouraged in this specific setting.

Taken into account both the relevant role of surgery in terms of effective cure for patients with NELM and the high incidence of recurrence following surgery, improvement in patient selection is necessary. The majority of the patients tend to recur within the liver and an aggressive surgical treatment (i.e., repeat hepatectomy) has been reported to be safe and effective. Liver resection should be reserved, in first instance, to patients with (I) good performance status; (II) absence of relevant co-morbidities; (III) no or minimum extra-hepatic disease, thus absence of peritoneal carcinomatosis; (IV) well-differentiated tumors, mainly G1-G2 tumors. However, radically intended surgery might be considered as a possible therapeutic option for highly selected stage IV GEP-NENs G3 (5).

In order to reduce the risk of recurrence, there is increasing interest on neoadjuvant and adjuvant options, even if strong evidence is still lacking. Current guidelines do not recommend any adjuvant treatment after curative resection and there is an urgent need for the identification of prognostic factors of tumor recurrence after radical surgery in order to select a specific subgroup of patients who might benefit from an adjuvant treatment.

Neoadjuvant therapy can convert unresectable tumors into resectable forms and reduce the recurrence rates by shrinking the tumor size and destroying micrometastatic lesions. The benefits of neoadjuvant therapy are well recognized in different digestive system exocrine tumors, but data on the actual role of neoadjuvant treatment in the specific setting of metastatic GEP-NENs are scanty. There is growing evidence that patients with NELM can achieve further clinical benefits from neoadjuvant therapy compared to patients who undergo surgery upfront, particularly through a reduction in tumor diameter and lymph node metastasis rate. Pre-operative 5-fluorouracil, doxorubicin and streptozocin regimen has been associated with improved OS compared to surgery alone among patients with advanced synchronous pancreatic NELM (1). The orally-available regimen capecitabine and temozolomide (CAPTEM) is associated with favorable radiographic objective response rates for locally advanced or metastatic pancreatic NENs and may facilitate selection of patients appropriate for surgical resection (6). For what matters the potential role of PRRT in the neoadjuvant setting little is known in the specific context of NELM. In a recent retrospective case series (7), PRRT lead to resectability of NELM in all the four included cases, being all the patients tumor free for 34.3 months on average after surgery with one patient experiencing a complete response after 4 years of follow-up. However, prospective studies are needed to confirm these preliminary considerations.

In summary, the management of NELM is challenging and should be addressed by tertiary centers in a multidisciplinary setting in order to get the best long-term outcomes. The advances in genetic and epigenetic sciences may guide the application of novel approaches, including neoadjuvant or adjuvant targeted options.

\section{Acknowledgments}

Funding: None.

\section{Footnote}

Provenance and Peer Review: This article was commissioned by the editorial office of Hepatobiliary Surgery and Nutrition. The article did not undergo external peer review.

Conflicts of Interest: The author has completed the ICMJE uniform disclosure form (available at https://hbsn. amegroups.com/article/view/10.21037/hbsn-21-178/coif). The author has no conflicts of interest to declare.

Ethical Statement: The author is accountable for all aspects of the work in ensuring that questions related to the accuracy or integrity of any part of the work are appropriately investigated and resolved.

Open Access Statement: This is an Open Access article distributed in accordance with the Creative Commons Attribution-NonCommercial-NoDerivs 4.0 International License (CC BY-NC-ND 4.0), which permits the noncommercial replication and distribution of the article with the strict proviso that no changes or edits are made and the original work is properly cited (including links to both the formal publication through the relevant DOI and the license). See: https://creativecommons.org/licenses/by-nc-nd/4.0/.

\section{References}

1. Cloyd JM, Ejaz A, Konda B, et al. Neuroendocrine liver 
metastases: a contemporary review of treatment strategies. Hepatobiliary Surg Nutr 2020;9:440-51.

2. Mazzaferro V, Sposito C, Coppa J, et al. The long-term benefit of liver transplantation for hepatic metastases from neuroendocrine tumors. Am J Transplant 2016;16:2892-902.

3. Ruzzenente A, Bagante F, Bertuzzo F, et al. Liver resection for neuroendocrine tumor liver metastases within Milan criteria for liver transplantation. J Gastrointest Surg 2019;23:93-100.

4. Sposito C, Rossi RE, Monteleone M, et al. Postrecurrence Survival After Liver Transplantation for Liver Metastases From Neuroendocrine Tumors.

Cite this article as: Rossi RE. Neuroendocrine liver metastases treatment strategies: a viewpoint. HepatoBiliary Surg Nutr 2021;10(4):561-563. doi: 10.21037/hbsn-21-178
Transplantation 2021. [Epub ahead of print]. doi: 10.1097/ TP.0000000000003802.

5. Merola E, Falconi M, Rinke A, et al. Radical intended surgery for highly selected stage IV neuroendocrine neoplasms G3. Am J Surg 2020;220:284-9.

6. Squires MH, Worth PJ, Konda B, et al. Neoadjuvant Capecitabine/Temozolomide for Locally Advanced or Metastatic Pancreatic Neuroendocrine Tumors. Pancreas 2020;49:355-60.

7. Chiapponi C, Lürssen N, Cremer B, et al. Peptide receptor radionuclide therapy as a two-step strategy for initially unresectable liver disease from neuroendocrine tumors: a single-center experience. Endocrine 2020;70:187-93. 